\title{
The PDE10A Inhibitor TAK-063 Reverses Sound-Evoked EEG Abnormalities in a Mouse Model of Fragile X Syndrome
}

\author{
Carrie R. Jonak $^{1}$ - Manbir S. Sandhu ${ }^{1} \cdot$ Samantha A. Assad ${ }^{1} \cdot$ Jacqueline A. Barbosa ${ }^{1} \cdot$ Mahindra Makhija $^{2}$. \\ Devin K. Binder ${ }^{1,3}$ (B)
}

Accepted: 6 January 2021 / Published online: 16 February 2021

(C) The American Society for Experimental NeuroTherapeutics, Inc. 2021, corrected publication 2021

\begin{abstract}
Fragile X syndrome (FXS) is a genetic neurodevelopmental syndrome characterized by increased anxiety, repetitive behaviors, social communication deficits, delayed language development, and abnormal sensory processing. Recently, we have identified electroencephalographic (EEG) biomarkers that are conserved between the mouse model of FXS (Fmrl KO mice) and humans with FXS. In this study, we test a specific candidate mechanism for engagement of multielectrode array (MEA) EEG biomarkers in the FXS mouse model. We administered TAK-063, a potent, selective, and orally active phosphodiesterase 10A (PDE10A) inhibitor, to Fmrl KO mice, and examined its effects on MEA EEG biomarkers. We demonstrate significant dose-related amelioration of inter-trial phase coherence (ITPC) to temporally modulated auditory stimuli by TAK-063 in Fmr1 KO mice. Our data suggest that TAK-063 improves cortical auditory stimulus processing in Fmrl KO mice, without significantly depressing baseline EEG power or causing any noticeable sedation or behavioral side effects. Thus, the PDE10A inhibitor TAK-063 has salutary effects on normalizing EEG biomarkers in a mouse model of FXS and should be pursued in further translational treatment development.
\end{abstract}

Key Words Fragile X syndrome · electroencephalography · biomarker · TAK-063 $\cdot$ phosphodiesterase

$\begin{array}{ll}\text { Abbreviations } \\ \text { EEG } & \text { Electroencephalography } \\ \text { FMRP } & \text { Fragile X mental retardation protein } \\ \text { FXS } & \begin{array}{l}\text { Fragile X syndrome } \\ \text { LC/MS/MS }\end{array} \\ & \begin{array}{l}\text { Liquid chromatography/tandem } \\ \text { mass spectrometry }\end{array} \\ \text { ITPC } & \begin{array}{l}\text { Inter-trial phase coherence } \\ \text { (phase locking factor) }\end{array} \\ \text { K/X } & \text { Ketamine/xylazine } \\ \text { MEA } & \text { Multielectrode array } \\ \text { PDE } & \text { Phosphodiesterase } \\ \text { PK } & \text { Pharmacokinetics }\end{array}$

Devin K. Binder dbinder@ucr.edu

1 Division of Biomedical Sciences, School of Medicine, University of California, 900 University Avenue, Riverside, CA 92521, USA

2 Takeda International - UK, Rare Diseases Therapeutic Area Unit, 1 Kingdom Street, London W2 6BD, UK

3 Neuroscience Graduate Program, University of California, Riverside, CA, USA

\section{Introduction}

Fragile X syndrome (FXS) is the most common genetic cause of intellectual disability with symptoms that overlap with autism spectrum disorders (ASDs) [1]. FXS is caused by a mutation in the Fragile X Mental Retardation 1 (Fmrl) gene and a loss of fragile X mental retardation protein (FMRP) [2]. FMRP is an RNA-binding protein that regulates synaptic function through regulation of protein translation [3]. Symptoms associated with FXS include increased anxiety, repetitive behaviors, social communication deficits, delayed language development, and abnormal sensory processing [4-13]. Abnormal sensory processing in FXS includes hypersensitivity and reduced habituation to repeated sensory stimuli $[14,15]$.

Identification of comparable biomarkers in humans and validated animal models is a critical step in facilitating preclinical to clinical therapeutic pipelines to treat neurodevelopmental disorders [16]. To provide relevant electrophysiological biomarkers, we have developed and applied multielectrode array (MEA) analysis in Fmrl KO mice, the mouse model of FXS $[17,18]$. Our system involves stable 
chronic in vivo implantation of a planar multielectrode array (MEA) on the surface of the mouse skull and enables lownoise 30-channel simultaneous EEG, which can then be used for resting and stimulus-evoked EEG acquisition in awake, freely moving mice [17]. For auditory stimulation, we use paradigms analogous to those used in human studies [19] and have demonstrated robust MEA-derived phenotypes of altered resting EEG power, single-trial and train-related EEG power, and inter-trial phase coherence (ITPC) to auditory chirp stimuli in Fmrl KO mice [18].

In this study, we test a specific candidate mechanism for engagement of MEA EEG biomarkers in the FXS mouse model. TAK-063 (1-[2-fluoro-4-(1H-pyrazol-1-yl)phenyl]-5methoxy-3-(1-phenyl-1H-pyrazol-5-yl)-pyridazin-4(1H)-one) is a potent, selective, and orally active phosphodiesterase $10 \mathrm{~A}$ (PDE10A) inhibitor $[20,21]$ that has been developed and tested in translational and clinical trials in schizophrenia [22-25] and Huntington's disease [26]. Phosphodiesterase is the enzyme that breaks down the signaling molecule cyclic AMP, and low levels of cAMP have been found in fragile $\mathrm{X}$ patients $[27,28]$.

Recently, PDE inhibition has proved to be a fruitful strategy in FXS animal models. For example, PDE2A inhibitors ameliorate cAMP metabolism alterations and synaptic plasticity defects and improve social interactions in mouse and rat models of FXS [29]. Acute treatment with a PDE-4 inhibitor rescues the mGluR-dependent LTD phenotype in FXS mice in hippocampal slices [30]. Chronic treatment of adult Fmrl KO mice was shown to restore the level of mGluRdependent LTD to that observed in wild-type animals. Further consolidation of the role of cAMP in FXS has been demonstrated by recently published data by Tetra Discovery Partners and FRAXA Research Foundation. BPN14770, a negative allosteric modulator of PDE4D, was shown to improve behavioral phenotypes in Fmrl KO mice (reduction of hyperarousal and improvement in nesting, marble burying and dendritic spine morphology) [31]. This compound is currently in a phase 2 clinical trial in FXS patients (https:// tetratherapeutics.com/science/pipeline/). Thus, ample evidence exists that PDE inhibition may be therapeutic in FXS. However, inhibition of PDE10A specifically has not been tested yet in animal models of FXS. In this study, we administer TAK-063, the specific orally active PDE10A inhibitor, to Fmrl $\mathrm{KO}$ mice, and examine the effects of TAK-063 on translational EEG biomarkers. A PDE10A inhibitor like TAK-063 is expected to have a better safety profile due to its high selectivity and restricted expression pattern within the CNS compared to PDE4 inhibitors. We demonstrate significant dose-related amelioration of inter-trial phase coherence (ITPC) to temporally modulated auditory stimuli by TAK-063 in Fmrl KO mice. Thus, the PDE10A inhibitor TAK-063 has salutary effects on normalizing EEG biomarkers in a mouse model of FXS.

\section{Methods}

\section{TAK-063}

TAK-063 was synthesized by Takeda Pharmaceutical Company Limited.

\section{Animals}

Male Fmr1 KO (B6.129P2-Fmr1tm1Cgr/J, stock \#003025) [32] and C57BL/6J WT (stock \#000664) mice were obtained from The Jackson Laboratory. Genotypes were confirmed by PCR analysis of genomic DNA isolated from mouse tails. Mice were maintained in an AAALAC accredited facility under a 12-h light/dark cycle and were provided irradiated rodent diet (PicoLab, 5053) and water ad libitum. All procedures were performed with approval from the University of California Animal Care and Use Committee and in accordance with the NIH Animal Care and Use Guidelines.

EEG recordings were obtained from $32 \mathrm{Fmrl} \mathrm{KO}$ and 8 WT mice. Male mice between 12 and 15 weeks of age were used for all EEG recordings.

\section{Study Design and Drug Treatment}

EEG responses were recorded 3-4 days after recovery from MEA implantation surgery and served as pre-drug baseline responses. After 14 days of daily oral administration of TAK-063 $(0.5$ and $5 \mathrm{mg} / \mathrm{kg})$ or vehicle $(0.5 \%$ methylcellulose), the same mice were recorded $2 \mathrm{~h}$ after treatment to determine post drug effects (Fig. 1). Fmrl KO mice received either $0.5 \mathrm{mg} / \mathrm{kg}$ TAK-063 $(n=12), 5 \mathrm{mg} / \mathrm{kg}$ TAK-063 $(n=$ $12)$, or vehicle $(n=8)$, and WT mice received vehicle $(n=8)$.

TAK-063 solutions were suspended using a vehicle of $0.5 \%(\mathrm{~m} / \mathrm{v})$ methylcellulose (Millipore Sigma, M0512) in distilled water. Stock solutions of TAK-063 were prepared every 8 days and stored at $4{ }^{\circ} \mathrm{C}$ until time of use. All drugs and vehicle solutions were administered with a sterile stainless steel curved oral gavage needle (Cadence Science, 7910). After each use, gavage needles were soaked in ethanol, rinsed with distilled water, and autoclaved.

\section{MEA Implantation}

Surgical and recording procedures were similar to those used in our recent publication [18]. Mice were anesthetized with isoflurane inhalation $(0.2-0.5 \%)$ and given an i.p. injection of $80 \mathrm{mg} / \mathrm{kg}$ ketamine (Zoetis, 10004027) and $10 \mathrm{mg} / \mathrm{kg}$ xylazine (Bimeda, 1XYL003). Mice were aseptically prepared for surgery and secured in a stereotaxic apparatus. Artificial tear ointment was applied to the eyes to prevent drying. Toe pinch reflex was used to measure anesthetic depth throughout the surgery, and supplemental doses of ketamine/ 


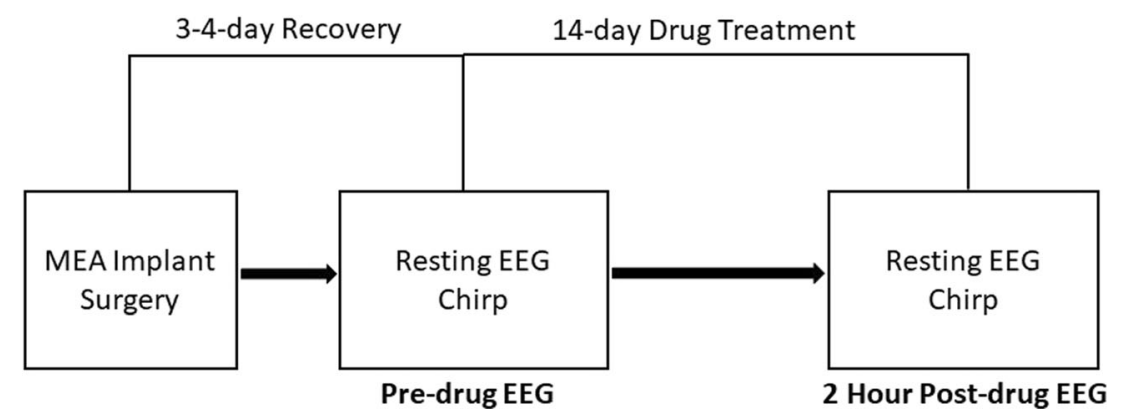

Fig. 1 Overview of the experimental design. After 4 days of recovery from MEA implantation, EEG responses were recorded and served as pre-drug baseline responses. After 14 days of daily TAK-063 (0.5 and

xylazine were administered as needed. Once the mouse was anesthetized, a midline sagittal incision was made along the scalp to expose the skull. A cotton-tip applicator was used to remove the periosteum from the skull and to clean the skull with saline. A surgical marker was used to mark bregma and positions of three screws. A dental drill was used to drill 1$\mathrm{mm}$-diameter holes in the skull overlying the left frontal cortex, left cerebellum, and right cerebellum. Screws (PlasticsOne, 00-96 X 1/16) were advanced into drilled holes until secure; special care was taken not to advance the screws beyond the point of contact with the dura. The probe grounding wire was placed in the nuchal musculature and the probe was placed on the skull surface carefully aligning the " + " in the center of the probe with bregma. Saline was added to the top of the probe to aid in adherence to the skull surface and allowed to dry. A 4-0 silk tie was used to secure the probe ribbon between the two cerebellum screws, and Teflon/plastic wrap was placed on top of the probe. Dental cement (Kuraray, 3382KA) was applied around the screws, on the base of the cotton-tip applicator post, and on the Teflon/plastic wrap covering the probe. Waterproof medical tape was used to secure the cotton-tip applicator to the probe connector. Triple antibiotic was applied along the edges of the dental cement followed by a subcutaneous injection of $0.1 \mathrm{mg} / \mathrm{kg}$ buprenorphine (Reckitt \& Colman, 5053624). Mice were placed on a heating pad to aid in recovery from anesthesia, and additional doses of buprenorphine were administered every $6-8 \mathrm{~h}$ for continuous analgesia during the first $48 \mathrm{~h}$ after surgery. Mice were then individually housed with nesting material (Ancare, NES3600), returned to the vivarium, and monitored daily until the day of EEG recordings.

\section{Acoustic Stimulation}

All experiments were conducted in a sound-attenuated chamber lined with anechoic foam (Gretch-Ken Industries, Oregon). Acoustic stimuli were generated using RPVDSEX software and RZ6 hardware (Tucker Davis Technologies, FL) and presented through a free-field speaker (MF1 Multi-Field Magnetic Speaker; Tucker-Davis Technologies, FL) located
$5 \mathrm{mg} / \mathrm{kg}$ p.o.) or vehicle treatment, the same mice were recorded $2 \mathrm{~h}$ after drug/vehicle to determine post-drug effects on EEG

12 in. directly above the cage. Sound pressure level (SPL) was modified using programmable attenuators in the RZ6 system. The speaker output was $\sim 70 \mathrm{~dB}$ SPL at the floor of the recording chamber with fluctuation of $\pm 3 \mathrm{~dB}$ for frequencies between 5 and $35 \mathrm{kHz}$ as measured with a $1 / 4$ inch Bruel and Kjaer microphone.

After 5 min of EEG recording without any sound (resting EEG), we used an acoustic stimulation paradigm that has been used in humans with FXS to enhance translational relevance. Ethridge et al. used a chirp-modulated tone (henceforth, "chirp") to induce synchronized oscillations in their EEG recordings [19]. The chirp stimulus used was broadband noise whose amplitude was modulated by a sinusoid with linearly increasing frequencies from 1 to $100 \mathrm{~Hz}$ [33-35]. Each stimulus was $2 \mathrm{~s}$ in duration, and the depth of modulation was $100 \%$. To avoid onset responses contaminating phase locking to the amplitude modulation of the chirp, the stimulus was ramped in sound level from 0 to $100 \%$ over $1 \mathrm{~s}$ (rise time) which then smoothly transitioned into chirp modulation of the noise. Chirp trains were presented 300 times each with the interval between each train randomly generated to be between 1 and $1.5 \mathrm{~s}$.

The chirp facilitates a rapid measurement of transient oscillatory entrainment (delta to gamma frequency range) to auditory stimuli of varying frequencies and can be used to compare oscillatory responses in different groups in clinical and pre-clinical settings [35]. Inter-trial phase coherence analysis (phase locking factor) [36] can then be used to determine the ability of neural generators to synchronize oscillations to the frequencies present in the repeated stimulus.

\section{Electrophysiology}

Resting and sound-evoked EEG recordings were obtained using the SmartBox (NeuroNexus) acquisition system from awake and freely moving mice $[17,18]$. A headstage and tether was connected to the probe post of the mouse skull (implanted during surgery) under brief isoflurane anesthesia. The mouse was then placed inside a grounded Faraday cage after recovery from isoflurane. This tether was then connected 


\section{WT Vehicle}

a

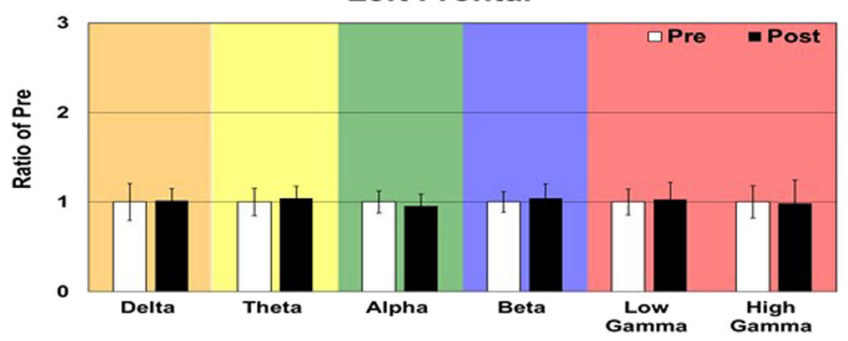

C

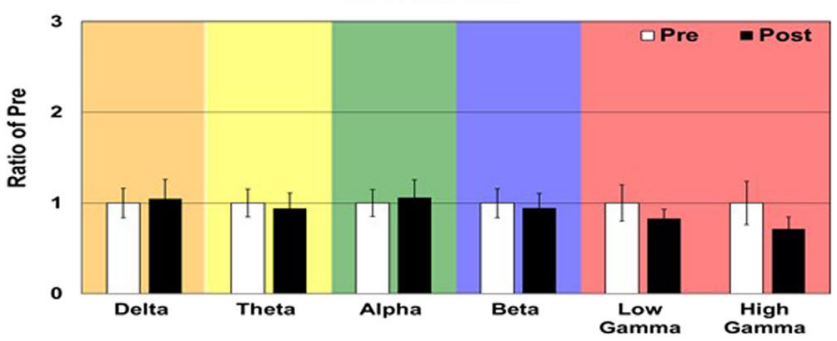

e

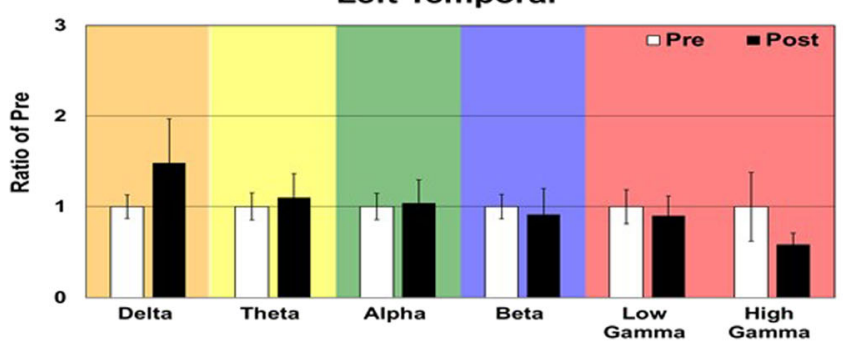

b

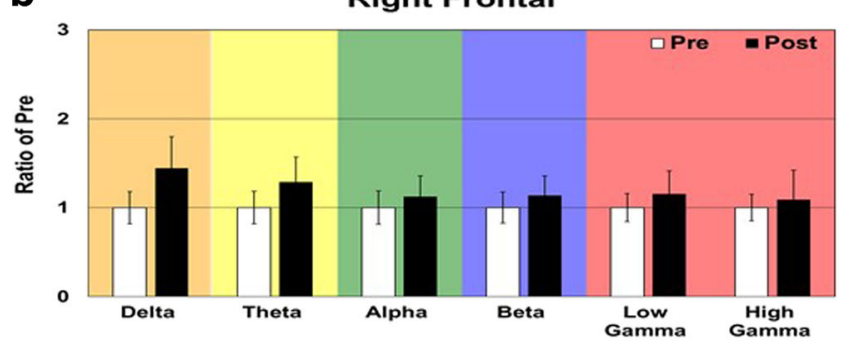

d

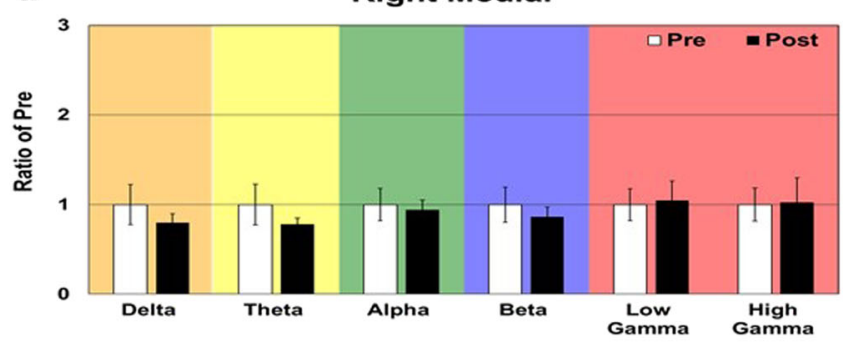

f

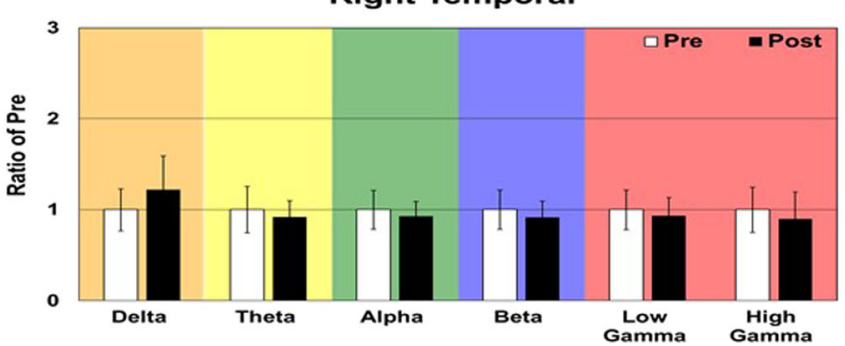

Fig. 2 Ratio of WT Post vehicle to Pre vehicle EEG power across frequency bands for distinct cortical regions (A-F). Values below 1 indicate lower EEG power in post vehicle treatment (Post) compared with pre vehicle treatment (Pre). * $p<0.05 ; * * p<0.01 . n=8$ Pre, $n=8$ Post

to a commutator located directly above the cage. Mice were allowed to habituate to being connected to the tether for 20 min before EEG recordings were obtained.

The SmartBox acquisition system was connected to the commutator to which the animal was attached. Acquisition hardware was set to lower $(0.5 \mathrm{~Hz})$ and upper $(500 \mathrm{~Hz})$ filters and data were sampled at a rate of $1250 \mathrm{~Hz}$. Sound delivery was synchronized with EEG recordings using a TTL pulse to mark the onset of each sound in a train. Five minutes of resting EEG was recorded in which no auditory stimuli were presented. This was followed by sound-evoked EEG recordings in response to chirp stimuli. After these experiments were completed, mice were euthanized with $5 \%$ isoflurane and brains and plasma samples were collected for drug exposure analysis for both TAK-063-treated cohorts.

\section{EEG Data Analysis}

Data analyses were performed using a combination of Analyzer 2.1 (Brain Vision Inc.), MATLAB, and SPSS.
Data were extracted from the Smartbox files and saved in a file format compatible with Analyzer 2.1 software. Data were first down sampled to $625 \mathrm{~Hz}$ and a $60-\mathrm{Hz}$ notch filter was used. EEG artifacts were removed using a semiautomatic procedure in Analyzer 2.1 for all recordings. Less than $20 \%$ of data were rejected due to artifacts from any single mouse.

Resting (no auditory stimulus) EEG data were divided into 1-s segments and Fast Fourier Transforms (FFT) was run on each segment using $10 \%$ Hanning window at $0.5-\mathrm{Hz}$ bin resolution and then average power $\left(\mu \mathrm{V} / \mathrm{Hz}^{2}\right)$ was calculated for each mouse from 1 to $100 \mathrm{~Hz}$. Power was then further binned into standard frequency bands: delta $(1-4 \mathrm{~Hz})$, theta $(4-8 \mathrm{~Hz})$, alpha $(8-13 \mathrm{~Hz})$, beta $(13-30 \mathrm{~Hz})$, low gamma $(30-55 \mathrm{~Hz})$, and high gamma $(65-100 \mathrm{~Hz})$.

Chirp trains were processed with Morlet wavelets linearly spaced from 1 to $100 \mathrm{~Hz}$ using voltage $(\mu \mathrm{V})$, and wavelet coefficients were exported as complex values for use with inter-trial phase coherence (ITPC) analysis. Wavelets were run with a Morlet parameter of 10 . This parameter was chosen since studies in humans found most robust difference around 


\section{KO Vehicle}

a

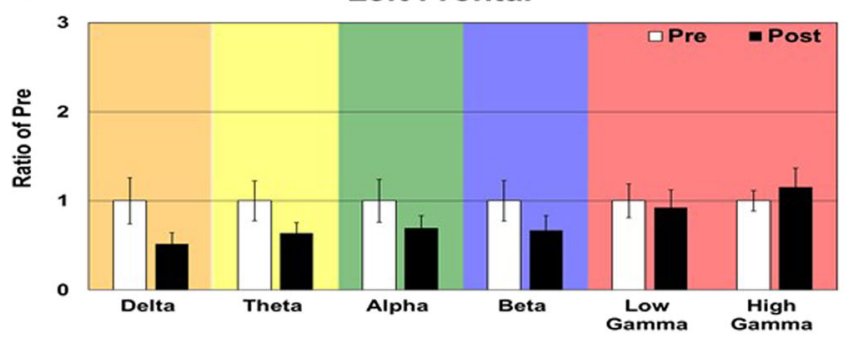

C

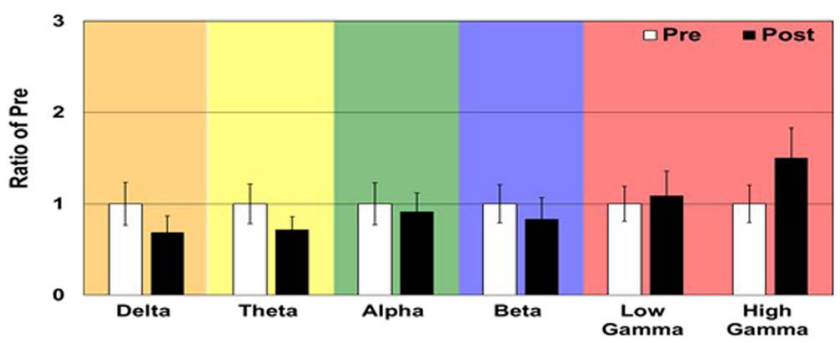

e

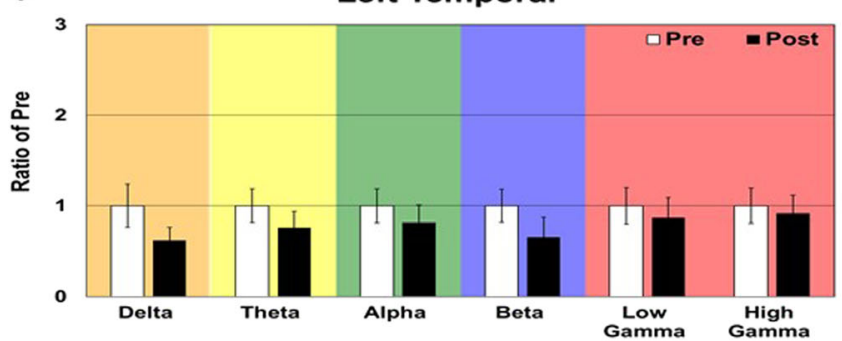

b

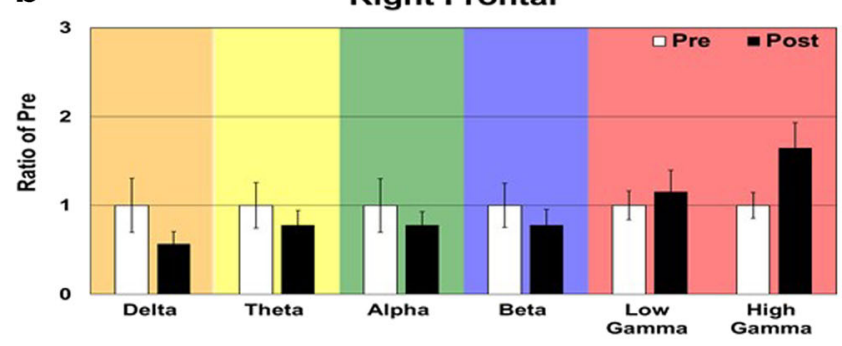

d

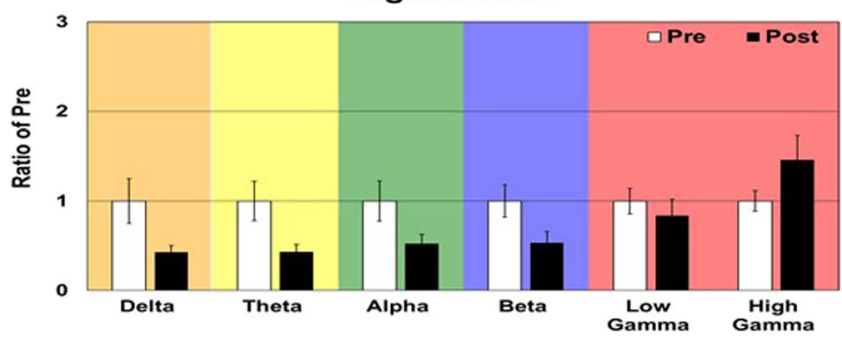

$\mathbf{f}$

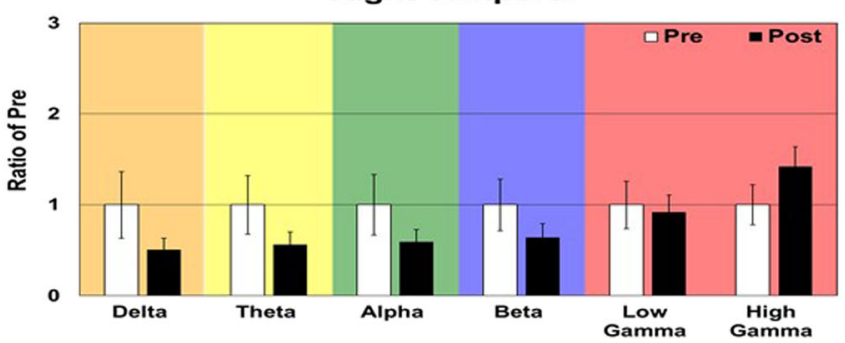

Fig. 3 Ratio of Fmrl KO Post vehicle to Pre vehicle EEG power across frequency bands for distinct cortical regions (A-F). Values below 1 indicate lower EEG power in post vehicle treatment (Post) compared with pre vehicle treatment (Pre). $* p<0.05 ; * * p<0.01 . n=8$ Pre, $n=8$ Post

$40 \mathrm{~Hz}$, where this parameter is centered [19]. To measure phase synchronization at each frequency across trials, intertrial phase coherence (ITPC) was calculated. The equation used to calculate ITPC is:

$\operatorname{ITPC}(f, t)=\frac{1}{n} \sum_{k=1}^{n} \frac{F_{k}(f, t)}{\left|F_{k}(f, t)\right|}$

where $f$ is the frequency, $t$ is the time point, and $k$ is the trial number. Thus, $F_{k}(f, t)$ refers to the complex wavelet coefficient at a given frequency and time for the $k$ th trial. There were no less than 275 chirp trials (out of 300 ) for any given mouse after segments containing artifacts were rejected.

\section{EEG Statistical Analyses}

The term "resting" is used to indicate EEGs recorded in these mice without any specific auditory stimuli. The data were analyzed for 2 factors: treatment (Pre, Post) and frequency (delta to gamma) for the cortical regions (left frontal, right frontal, left medial, right medial, left temporal, and right temporal). We analyzed the raw data using two-way ANOVA. Data were expressed as ratio of pre-treatment (Pre) values to gauge relative differences in various factors using the same scale.

Statistical group comparisons of ITPC in chirp trains were quantified using a Monte Carlo permutation approach. Analysis was conducted by binning time into 256 parts and frequency into 100 parts, resulting in a $100 \times 256$ matrix. Non-parametric analysis was used to determine contiguous regions in the matrix that were significantly different from a distribution of 2000 randomized Monte Carlo permutations based on previously published methods [37]. Cluster sizes of the real treatment assignments (both positive and negative direction, resulting in a two-tailed alpha of $p=0.025$ ) that were larger than $97.25 \%$ of the random group assignments were considered significantly different between experimental conditions. This method avoids statistical assumptions about the data and corrects for multiple comparisons.

In all cases where treatment means are reported, SEM was used. In all cases, $p$ values $<0.05$ were considered significant 


\section{KO 0.5 mg/kg TAK-063}
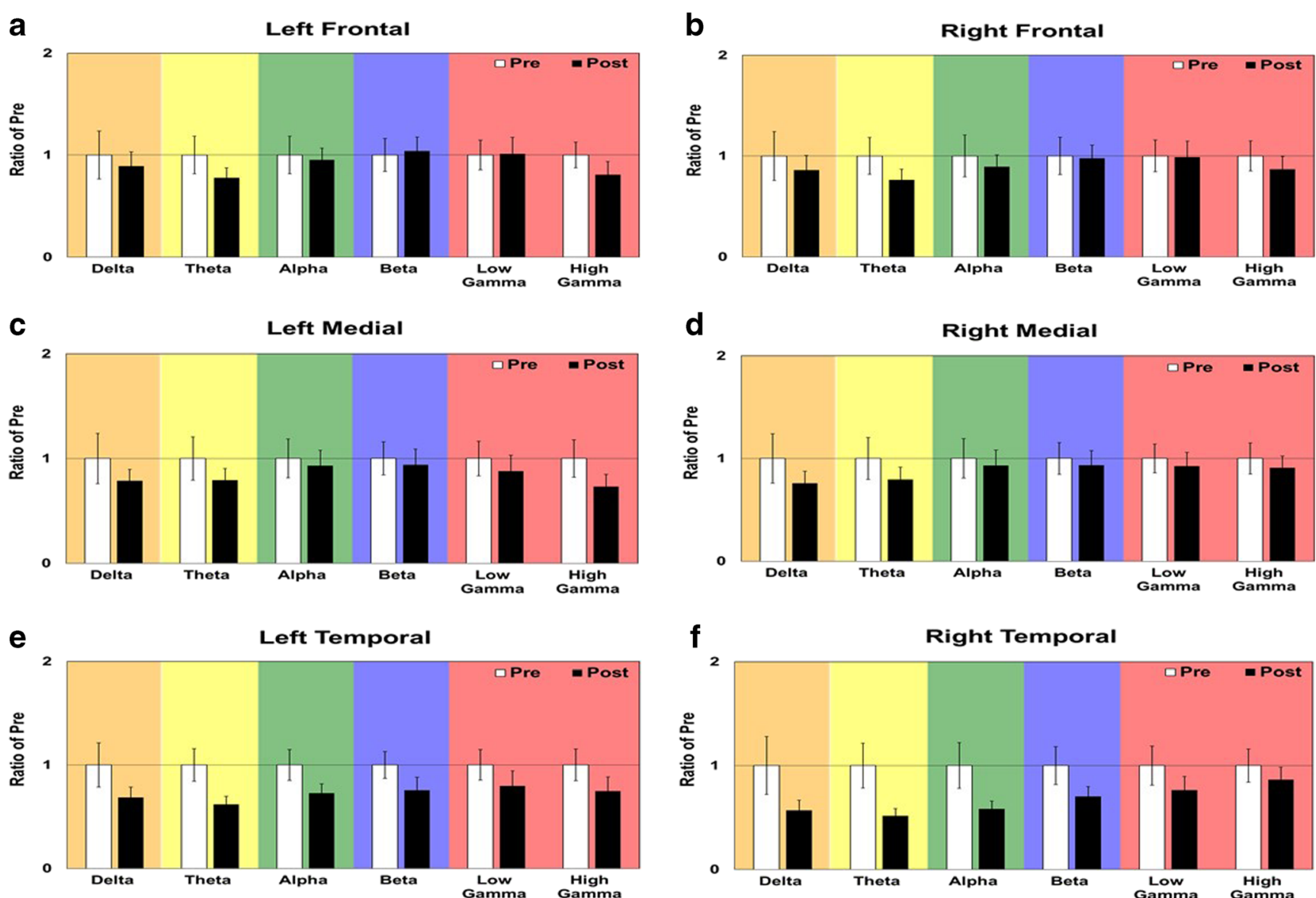

Fig. 4 Ratio of Fmrl KO Post $0.5 \mathrm{mg}$ TAK-063 to Pre EEG power across frequency bands for distinct cortical regions (A-F). Values below 1 indicate lower EEG power in post treatment (Post) compared with pre-treatment (Pre). $* p<0.05 ; * * p<0.01 . n=12$ Pre, $n=12$ Post

for ANOVA and Student's $t$ tests. Where $t$ tests were performed, $r$ was calculated as an effect size. When interactions were found and multiple comparisons for ANOVA were made, data were analyzed on each factor for simple effects and corrected for using Bonferroni adjusted $p$ values.

\section{Pharmacokinetic Analysis}

Plasma and brain samples were collected immediately after EEG recording and stored in $-80{ }^{\circ} \mathrm{C}$ until time of use for pharmacokinetic (PK) analysis. A $50-\mu \mathrm{L}$ aliquot of plasma and $20 \%$ brain tissue homogenate in saline were deproteinized by the addition of $150 \mu \mathrm{L}$ acetonitrile containing $500 \mathrm{ng} / \mathrm{mL}$ diclofenac sodium as an internal standard. After centrifugation, $60 \mu \mathrm{L}$ of supernatant was diluted with $160 \mu \mathrm{L}$ of a mixture of mobile phase A and B (7:3, v/v). Then, $40 \mu \mathrm{L}$ of the diluted solution was further diluted with $160 \mu \mathrm{L}$ of a mixture of mobile phase A and B (7:3, v/v) and applied to liquid chromatography/tandem mass spectrometry (LC/MS/MS).

Table 1 Plasma and brain concentrations of TAK-063 at $3 \mathrm{~h}$ after the repeated oral administration of TAK-063

\begin{tabular}{lllllllllllllllll}
\hline Dose & Tissue & \multicolumn{1}{l}{ Concentration (ng/mL or ng/g) } & \multicolumn{1}{c}{ Mean } & SD \\
\hline $0.5 \mathrm{mg} / \mathrm{kg}$ & Plasma & 163.1 & 169.7 & 201.2 & 45.6 & 69.5 & 61.6 & 37.8 & 238.0 & 53.5 & 67.0 & 52.3 & 63.1 & 101.9 & 70.2 \\
& Brain & 182.4 & 161.2 & 140.5 & 69.2 & 92.6 & 92.1 & 75.1 & 56.1 & 69.8 & 78.2 & 69.4 & 108.1 & 99.6 & 40.6 \\
& Brain/Plasma & 1.12 & 0.95 & 0.70 & 1.52 & 1.33 & 1.50 & 1.99 & 0.24 & 1.30 & 1.17 & 1.33 & 1.71 & 1.24 & 0.46 \\
$5 \mathrm{mg} / \mathrm{kg}$ & Plasma & 428.8 & 400.6 & 822.5 & 835.5 & 596.6 & 515.2 & 393.6 & 683.8 & 414.3 & 357.3 & 466.7 & 304.8 & 518.3 & 178.1 \\
& Brain & 239.2 & 141.1 & 247.3 & 248.5 & 274.5 & 213.3 & 275.0 & 214.4 & 182.5 & 193.4 & 224.8 & 177.3 & 219.3 & 40.7 \\
& Brain/Plasma & 0.56 & 0.35 & 0.30 & 0.30 & 0.46 & 0.41 & 0.70 & 0.31 & 0.44 & 0.54 & 0.48 & 0.58 & 0.45 & 0.13 \\
& & & & & & & & & & & & & &
\end{tabular}




\section{KO 5 mg/kg TAK-063}
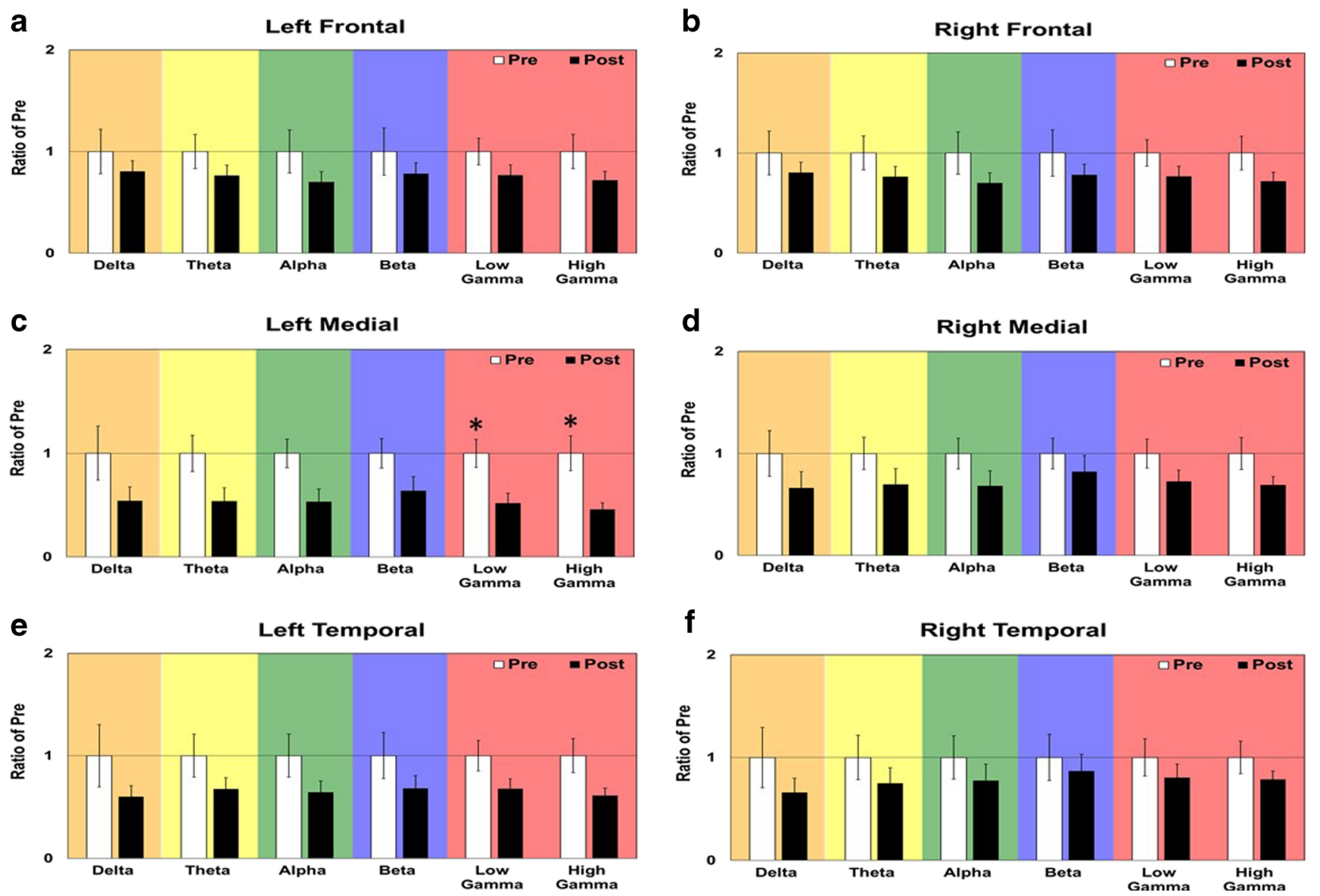

Fig. 5 Ratio of Fmrl KO Post $5 \mathrm{mg}$ TAK-063 to Pre EEG power across frequency bands for distinct cortical regions (A-F). Values below 1 indicate lower EEG power in post treatment (Post) compared with pre-treatment (Pre). * $p<0.05 ; * p<0.01 . n=12$ Pre, $n=12$ Post

Concentrations of TAK-063 in plasma and brain samples were quantified by LC/MS/MS analysis using a UFLC liquid chromatograph system (Shimadzu, Kyoto, Japan) and an API5000 mass spectrometer (SCIEX, Framingham, MA, USA). The liquid chromatography conditions were as follows: mobile phase A was a mixture of $10 \mathrm{mmol} / \mathrm{L}$ ammonium formate and formic acid (100:0.2, $\mathrm{v} / \mathrm{v})$, and mobile phase B was a mixture of acetonitrile and formic acid (100:0.2, v/v). The following gradient program was used at a flow rate of $0.7 \mathrm{~mL} / \mathrm{min}$ : $5 \%$ of B at $0-0.2 \mathrm{~min}$, linear gradient $5-99 \%$ of $B$ at $0.2-1.3 \mathrm{~min} ; 99 \%$ of $\mathrm{B}$ at $1.3-2.0 \mathrm{~min}$, linear gradient $99-5 \%$ of B at $2.0-2.01 \mathrm{~min} ; 5 \%$ of B at $2.01-2.6 \mathrm{~min}$. A Shimadzu Shim-pack XR-ODS column $(2.2 \mu \mathrm{m}$ particle size, $2.0 \times 30 \mathrm{~mm}$; Shimadzu) was used, and the column temperature was set to $50{ }^{\circ} \mathrm{C}$. The injection volume was $1 \mu \mathrm{L}$ for plasma and $3 \mu \mathrm{L}$ for brain. The mass spectrometer was operated in the positive electrospray ionization mode. A selective reaction monitoring method with transitions of $\mathrm{m} / \mathrm{z} 429.03 \rightarrow$ 188.06 for TAK-063 and $296.10 \rightarrow 214.20$ for diclofenac was adopted. Concentrations of the compound were determined by Analyst software (SCIEX), using the peak areas of the compound normalized to those of the internal standard in the LC/MS/MS chromatograms. The lower limit of quantification (LLOQ) of the compound in plasma was $3 \mathrm{ng} / \mathrm{mL}$, and that in brain was $15 \mathrm{ng} / \mathrm{mL}$. The brain-to-plasma concentration ratio of the compound in each animal was calculated using Microsoft Excel (Microsoft, Redmond, WA, USA).

\section{Results}

\section{Resting State Power in TAK-063-Treated Mice}

Before EEG recordings were acquired, mice were allowed to habituate to the recording chamber as well as being attached to the recording tether for a total of $20 \mathrm{~min}$. After mice were habituated, resting EEG was recorded for $5 \mathrm{~min}$ in the absence of any specific auditory stimulation. Resting EEG data were analyzed with two-way ANOVA for 2 factors: treatment (Pre, Post) and frequency (delta to gamma) in the cortical regions (left frontal, right frontal, left medial, right medial, left temporal, right temporal). There were no significant differences in frequency band 


\section{WT Vehicle}
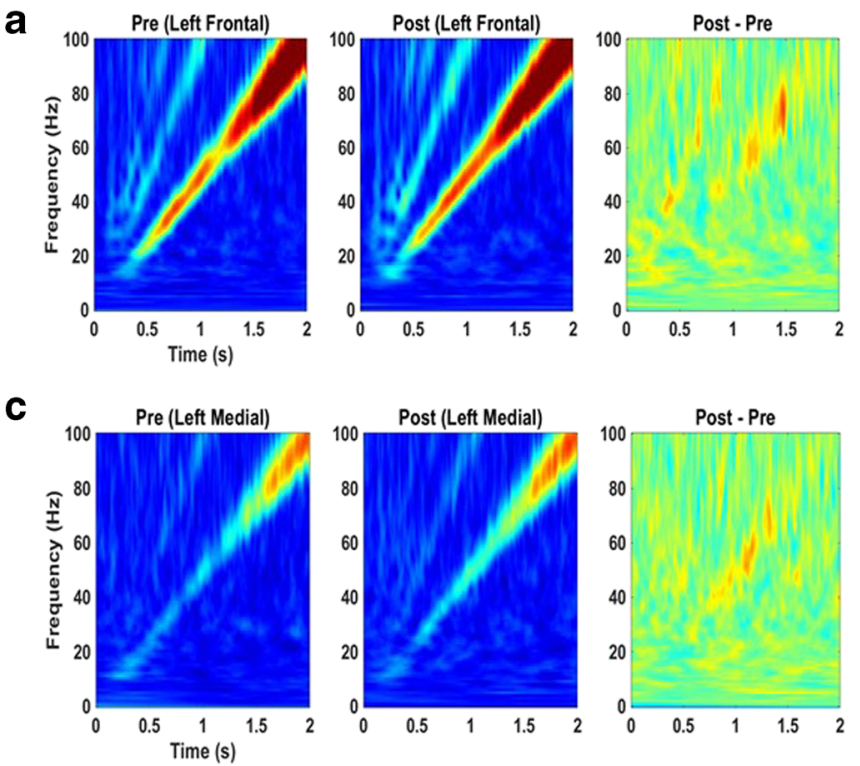

$\mathbf{e}$
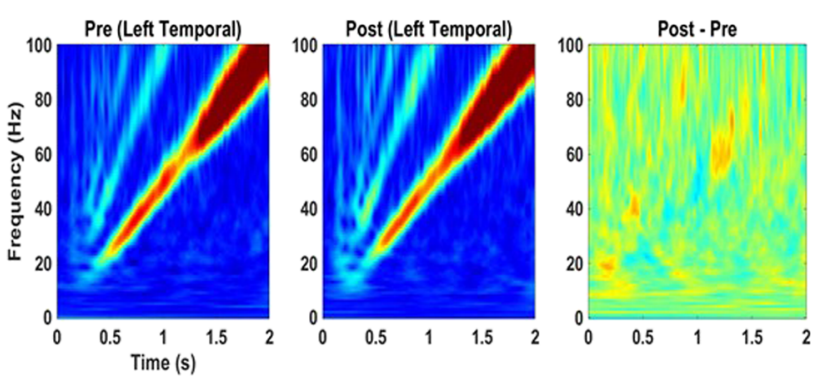

0

ITPC

Fig. 6 Auditory chirp stimulation in Pre vs. Post vehicle in WT mice. For each cortical region (A-F), the left panel shows the averaged inter-trial phase coherence (ITPC or phase locking factor) before vehicle treatment (Pre), the middle panel shows the averaged ITPC after vehicle treatment

power in the WT vehicle (Fig. 2), Fmrl KO vehicle (Fig. 3), and Fmrl KO 0.5 mg/kg TAK-063 (Fig. 4)-treated mice. However, at the higher dose of TAK-063 (5 mg/kg), significant decreases were observed in low and high gamma band power in the left medial cortical region of Fmrl KO mice with no significant differences in all other bands. These findings suggest that TAK-063 does not significantly impact resting EEG power.

\section{Increased Phase Locking to Temporally Modulated Stimuli in TAK-063-Treated Mice}

Inter-trial phase coherence (ITPC) (also called phase locking factor) measures the reliability of synchronization of neural responses to repetitions of the auditory chirp stimuli, with the EEG response entrained to the chirp modulation frequency. There were no significant ITPC differences with vehicle treatment in WT (Fig. 6) or Fmrl KO (Fig. 7) mice. In Fmrl KO mice treated with $0.5 \mathrm{mg} / \mathrm{kg}$ TAK-063, there were significant IPTC increases in all cortical regions other than the left and right medial areas
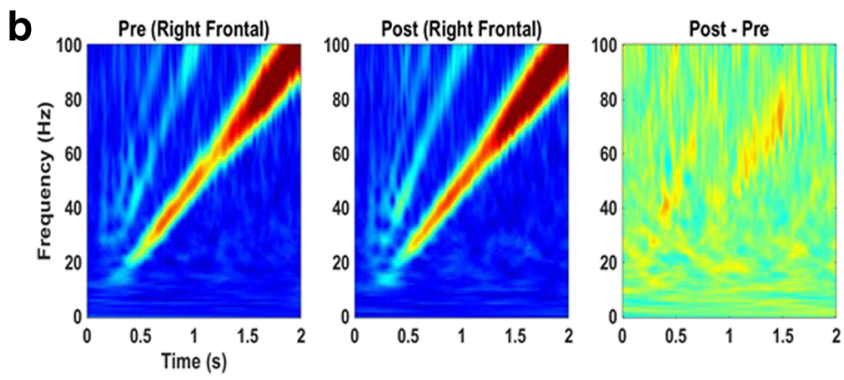

d
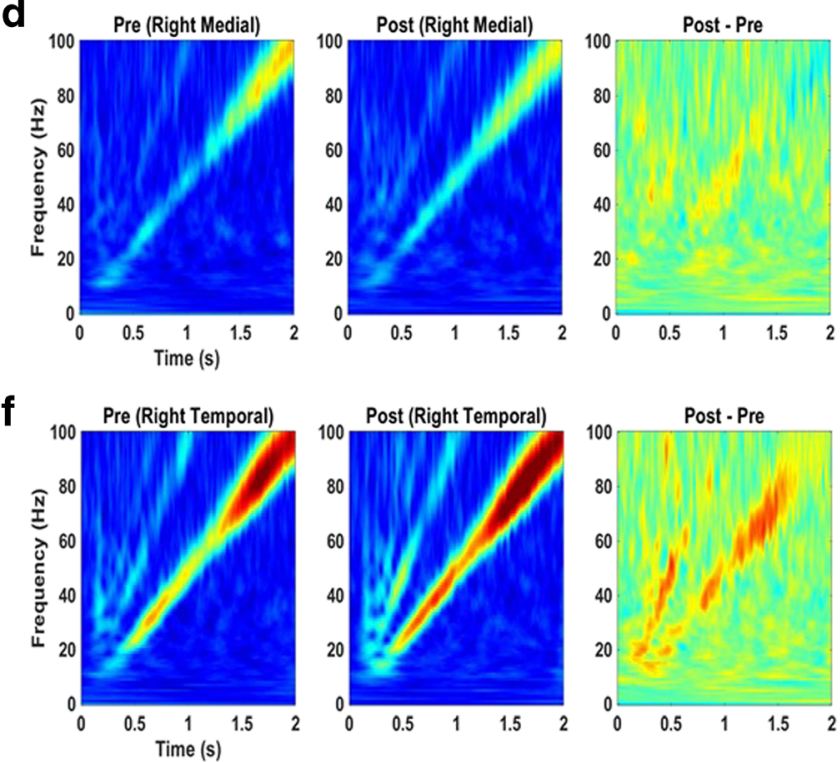

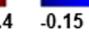

ITPC Difference $\quad 0.15$

(Post), and the right panel shows Post-Pre. Significant increases in ITPC in Post compared to Pre are shown in black-outlined areas. Red areas in the right panels (Post-Pre) represent positive ITPC differences and blue areas represent negative ITPC differences

(Fig. 8). Fmrl KO mice treated with $5 \mathrm{mg} / \mathrm{kg}$ TAK-063 also exhibited significant IPTC increases in all cortical regions other than the left and right medial areas (Fig. 9).

\section{Pharmacokinetic Dose Proportion and Exposure}

Pharmacokinetic (PK) analysis of plasma and brain samples revealed a dose-proportional and significant exposure in both periphery and CNS compartments (Table 1). Analysis of TAK-063 levels showed a mean plasma concentration of $101.9 \mathrm{ng} / \mathrm{ml}$ (SD 70.2) and $518.3 \mathrm{ng} / \mathrm{ml}$ (SD 178.1); and a mean brain tissue concentration of $99.6 \mathrm{ng} / \mathrm{g}$ (SD 40.6) and $219.3 \mathrm{ng} / \mathrm{g}$ (SD 40.7) for 0.5 and $5 \mathrm{mg} / \mathrm{kg}$ doses respectively. Comparison of these values to the in vivo $\mathrm{IC}_{50}$ of $1.1 \mathrm{ng} / \mathrm{ml}$ of TAK063 suggests that the plasma and brain concentrations remained above the target concentration and that penetration to the brain is such as to make pharmacodynamic effects a reasonable expectation. 


\section{KO Vehicle}
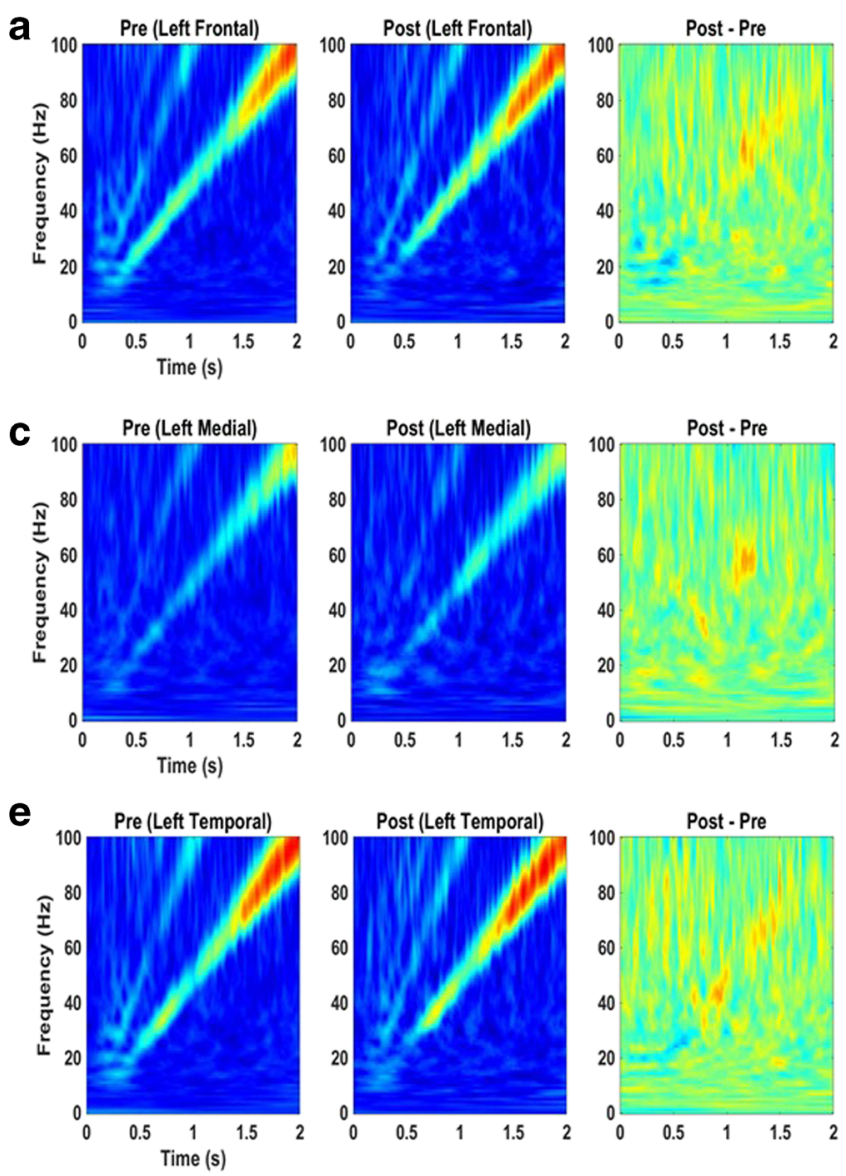

0

ITPC

Fig. 7 Auditory chirp stimulation in Pre vs. Post vehicle in Fmrl KO mice. For each cortical region (A-F), the left panel shows the averaged inter-trial phase coherence (ITPC or phase locking factor) before vehicle treatment (Pre), the middle panel shows the averaged ITPC after vehicle

\section{Discussion}

In this paper, we used 30-channel mouse skull surface MEA $[17,18]$ to test the effects of the orally active selective PDE10A inhibitor TAK-063 on translational EEG biomarkers in Fmrl KO mice. We tested TAK-063 at two doses $(0.5 \mathrm{mg} / \mathrm{kg}$ and $5 \mathrm{mg} / \mathrm{kg})$. First, we found that TAK-063 at either dose does not significantly alter overall resting EEG power (Figs. 4 and 5). However, the higher dose of TAK$063(5 \mathrm{mg} / \mathrm{kg})$ significantly reduced EEG gamma power in the left medial region of the brain (Fig. 5). Second, Fmrl KO mice exhibited a significant decrease in ITPC throughout all cortical regions as we have previously observed (Figs. 6 and 7) [18]. Third, TAK-063 $(0.5 \mathrm{mg} / \mathrm{kg}$ and $5 \mathrm{mg} / \mathrm{kg})$ increased ITPC in all cortical regions other than the left and right medial areas (Figs. 8 and 9). These findings show that TAK-063 is effective in increasing phase coherence to time-varying stimuli. These data also indicate region-specific differences on temporal processing with TAK-063 treatment.
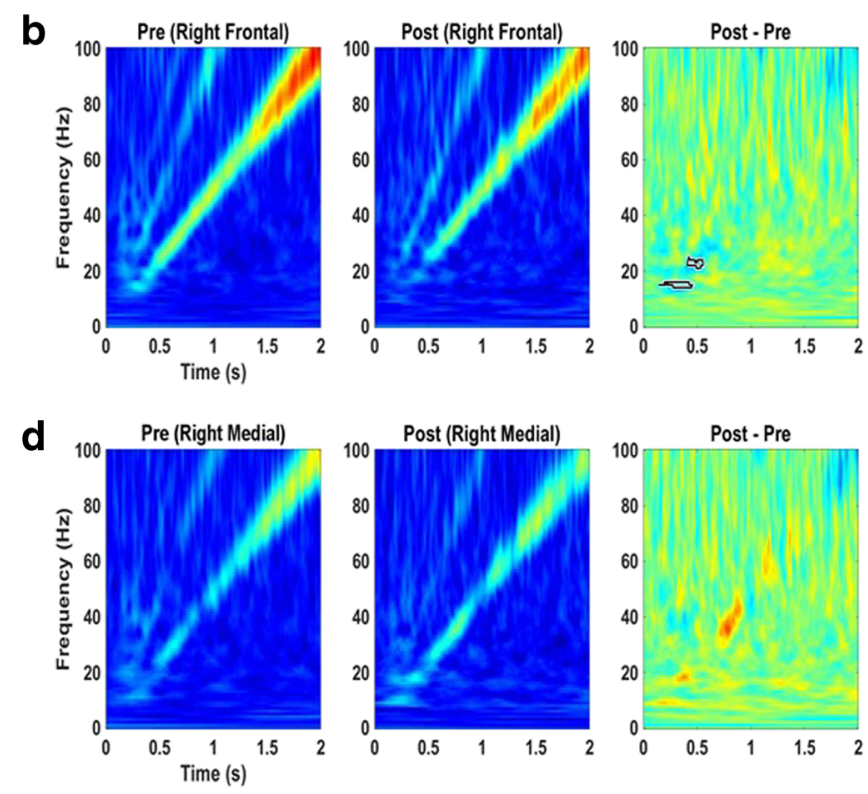

f
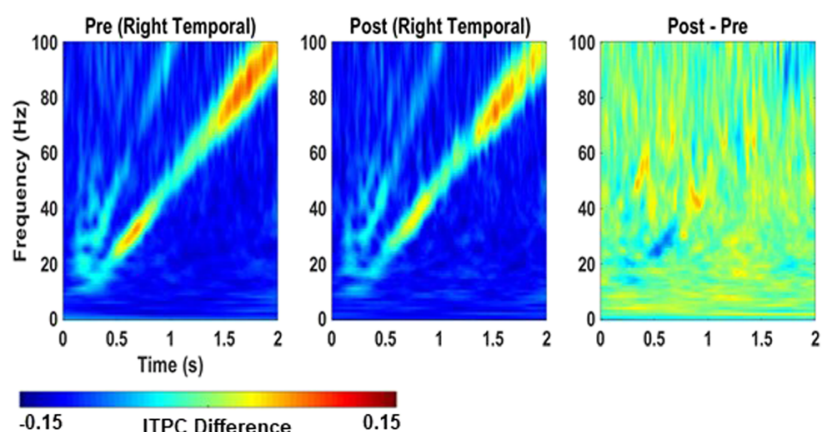

$-0.15$

ITPC Difference

treatment (Post), and the right panel shows Post-Pre. Significant increases in ITPC in Post compared to Pre are shown in black-outlined areas. Red areas in the right panels (Post-Pre) represent positive ITPC differences and blue areas represent negative ITPC differences

\section{PDE Inhibition as a Therapeutic Strategy in FXS}

Berry-Kravis and colleagues found reduced cAMP and altered cAMP metabolism in patients with FXS [27, 28]. The first report was a study of 14 patients with FXS compared to 21 control individuals; platelets from FXS patients had significantly lower cAMP production [28]. Subsequent studies confirmed this finding $[27,38]$. This led to the concept that PDE inhibition (which would be expected to increase levels of cAMP) might be a therapeutic approach in FXS. Recent studies strongly support this idea. Maurin and colleagues found that PDE2A inhibition in Fmrl KO mice rescued dendritic spine phenotypes and hippocampal mGluR-dependent longterm depression [29]. In addition, they found the PDE2A inhibition rescued social and communication deficits in both mouse and rat Fmrl KO animals [29]. PDE4 inhibition has also been demonstrated to rescue FXS phenotypes, in particular rescuing aberrant synaptic plasticity in Drosophila and mouse models of FXS [30]. Recently, it has also been shown 
KO 0.5mg TAK-063

a

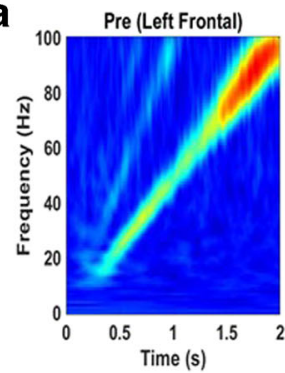

C

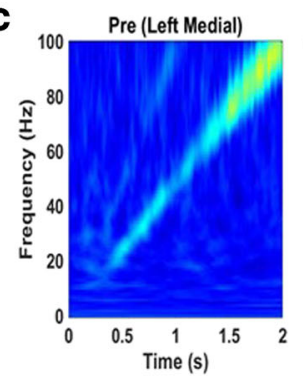

e
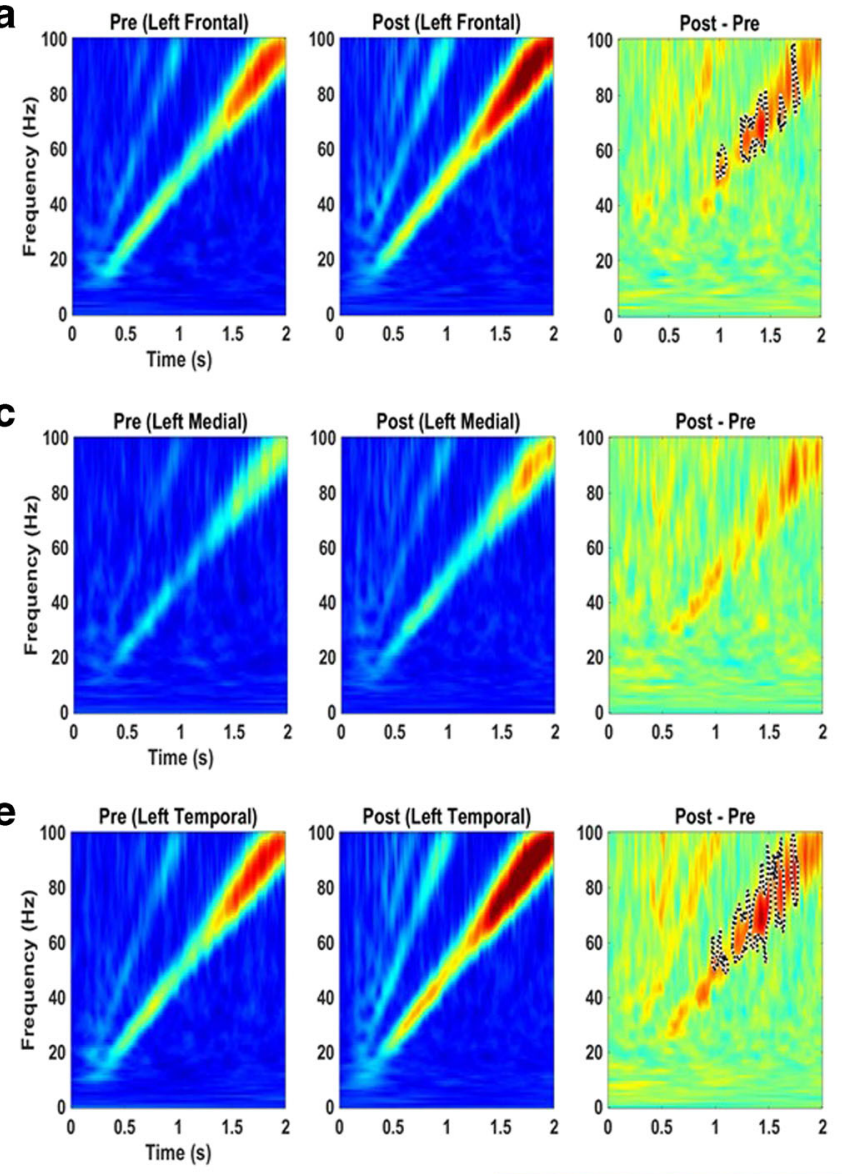

d
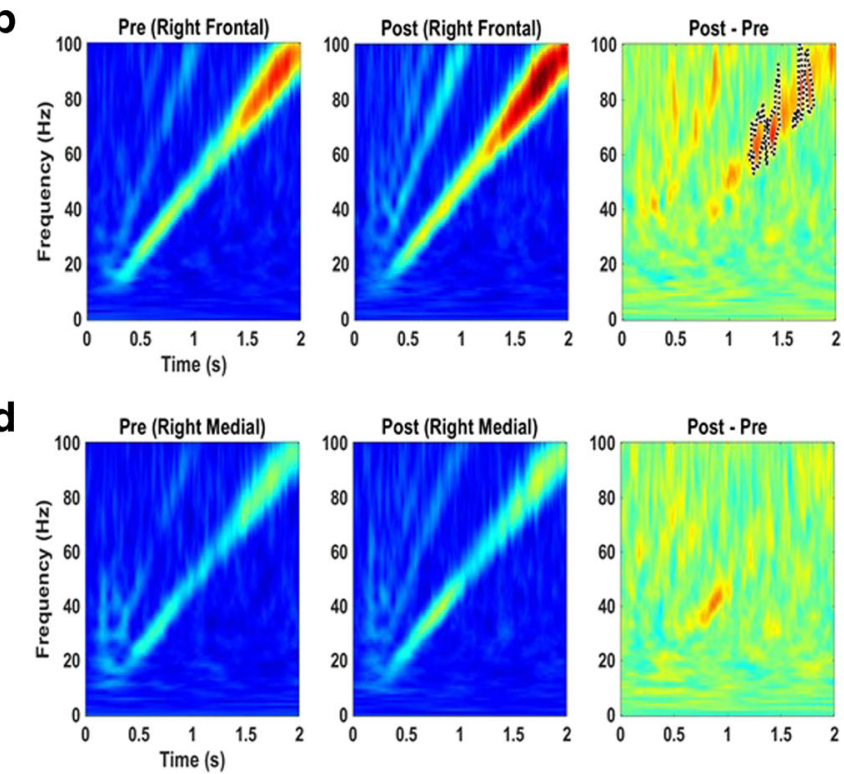

f

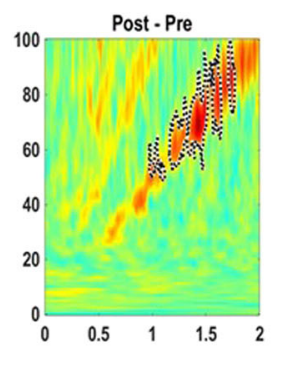

\section{政}
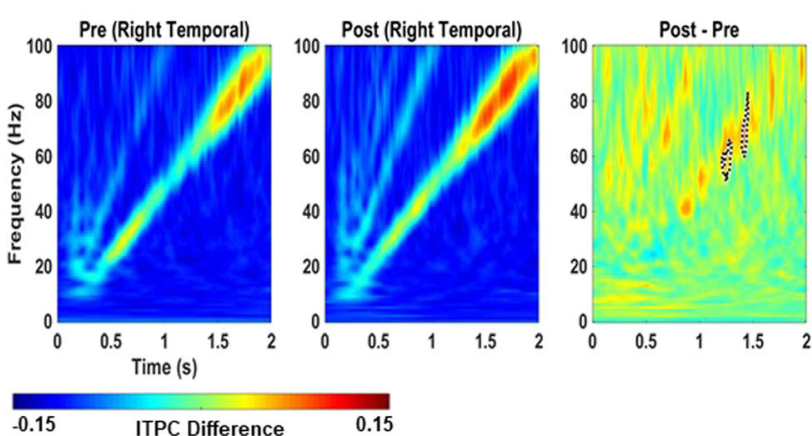

Fig. 8 Auditory chirp stimulation in Pre vs. Post $0.5 \mathrm{mg}$ TAK-063 in Fmrl KO mice. For each cortical region (A-F), the left panel shows the averaged inter-trial phase coherence (ITPC or phase locking factor) before drug treatment (Pre), the middle panel shows the averaged ITPC after drug treatment (Post), and the right panel shows Post-Pre. Significant increases in ITPC in Post compared to Pre are shown in black-outlined areas. Red areas in the right panels (Post-Pre) represent positive ITPC differences and blue areas represent negative ITPC differences that BPN14770, a negative allosteric modulator of PDE4D, reduces hyperarousal, improved social interaction, and improved nesting and marble burying behaviors as well as dendritic spine morphology [31]. This drug is now in a clinical trial (https://www.fraxa.org/fragile-x-clinical-trial-of-newpde4-inhibitor-from-tetra/).

\section{TAK-063, Selective PDE10A Inhibitor}

TAK-063 was developed as a potent (enzyme inhibition $\mathrm{IC}_{50}=$ $0.30 \mathrm{nM}$ ), selective (>15,000-fold over other PDEs), and orally active phosphodiesterase 10A (PDE10A) inhibitor [20, 21]. PDE10A is expressed primarily in the striatal medium spiny neurons (MSNs), which receive extensive cortical (glutamatergic), thalamic, and nigral (dopaminergic) input. So far, TAK-063 has been tested in translational and clinical trials in schizophrenia [22-25] and Huntington's disease [26]. TAK-063 has been tested in rats for its antipsychotic activity and side effects (hyperprolactinemia, hyperglycemia, catalepsy) and was found to be superior in terms of its tolerability profile compared to other antipsychotic agents such as haloperidol, olanzapine, and aripiprazole [21]. A phase 1 study with TAK-063 in Japanese and non-Japanese healthy volunteers indicated it was safe and well-tolerated at doses up to $1000 \mathrm{mg}$ with a PK profile supporting once daily dosing [39]. Given the tolerability profile and the known importance of PDE inhibition as a therapeutic strategy in FXS, this provided the impetus for the current study.

\section{Effect of TAK-063 on EEG and ITPC}

Importantly, administration of TAK-063 at the doses in this study $(0.5$ and $5 \mathrm{mg} / \mathrm{kg}$ ) did not lead to sedation or obvious side effects in the mice over the 2 -week study period. In fact, we observed very little effect of TAK-063 on resting EEG power (Figs. 4 and 5). This is in contrast to CNS depressant drugs at effective doses; for example, while racemic baclofen 


\section{KO 5mg TAK-063}
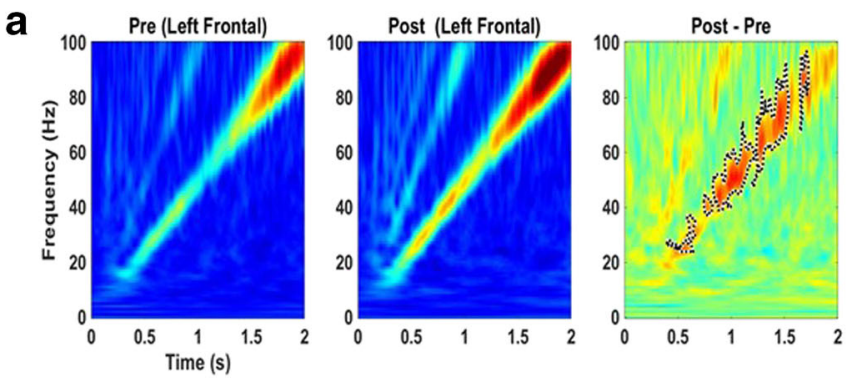

C
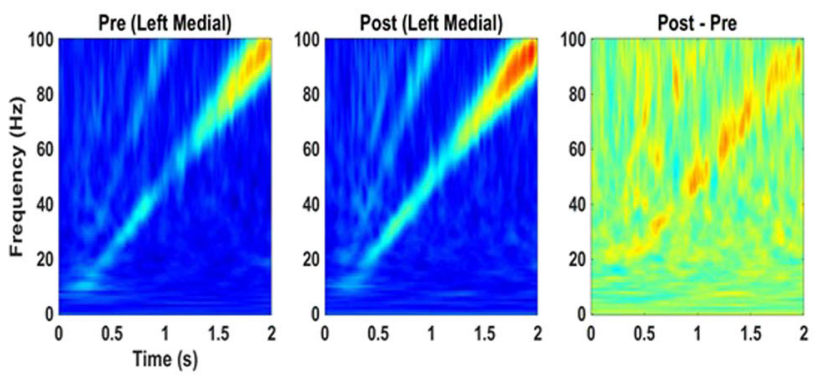

e
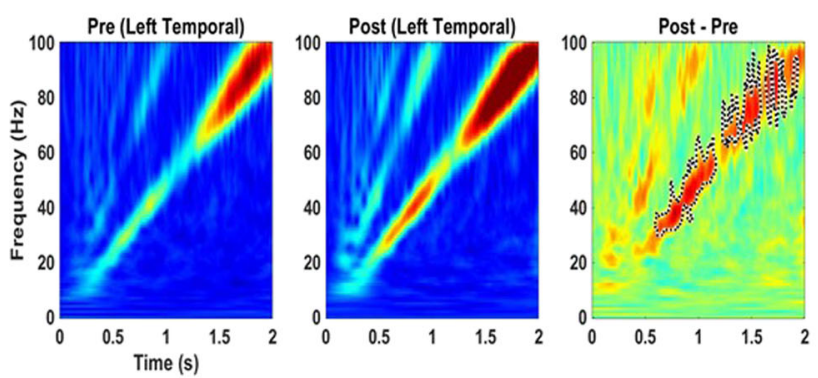

0 b

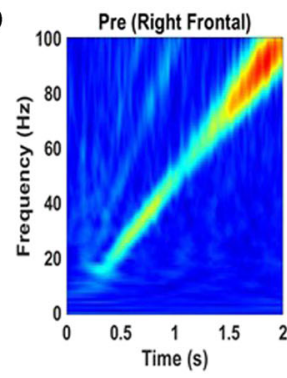

d

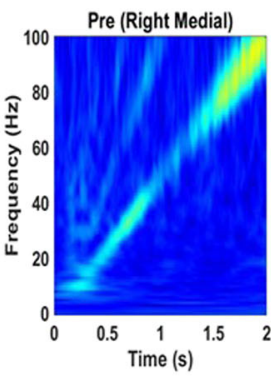

f

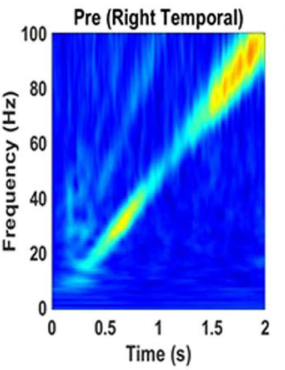

Post (Right Frontal)

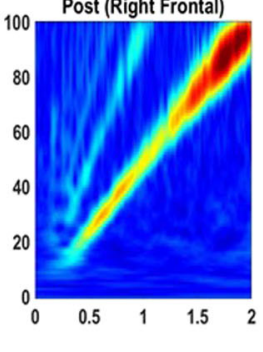

Post (Right Medial)

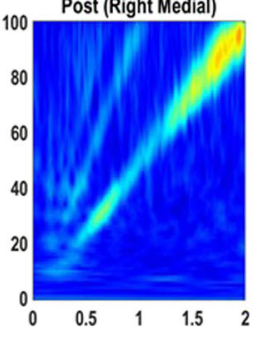

Post (Right Temporal)

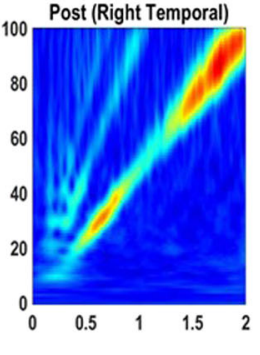

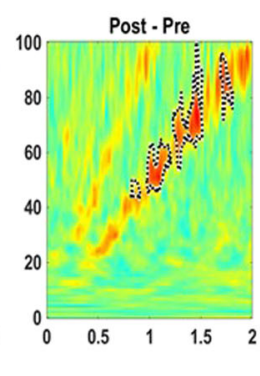

Post - Pre

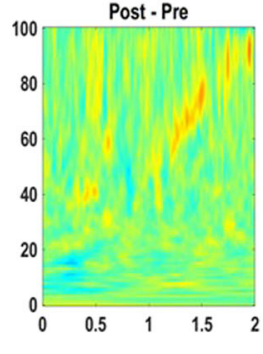

Post - Pre

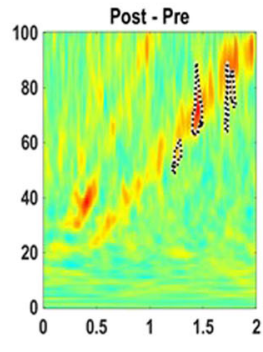

Fig. 9 Auditory chirp stimulation in Pre vs. Post 5 mg TAK-063 in Fmrl KO mice. For each cortical region $(\mathrm{A}-\mathrm{F})$, the left panel shows the averaged inter-trial phase coherence (ITPC or phase locking factor) before drug treatment (Pre), the middle panel shows the averaged ITPC after

drug treatment (Post), and the right panel shows Post-Pre. Significant increases in ITPC in Post compared to Pre are shown in black-outlined areas. Red areas in the right panels (Post-Pre) represent positive ITPC differences and blue areas represent negative ITPC differences had a therapeutic effect on reducing gamma power in EEG in Fmr1 KO mice, it nevertheless has had dose-limiting sedation effects in published studies [40]. In addition, in our hands, racemic baclofen has therapeutic effects on EEG and ITPC but only at sedating doses (Jonak et al., unpublished data). Therefore, a drug that improves FXS EEG biomarkers at nonsedating doses may be quite promising to develop therapeutically.

ITPC (inter-trial phase coherence or phase locking factor) measures the degree of synchronization of brain response to a presented stimulus. In our case, this is the auditory "chirp" stimulus identical to that used in human clinical trials [19]. FXS patients [19] and Fmrl KO mice [18] have impaired ITPC to auditory "chirp" stimuli (also compare Figs. 6 and 7). It is important to recognize that ITPC and other EEG biomarkers have recently been correlated to other relevant behavioral measures in FXS patients in larger groups [41]. TAK063 at either dose tested ( 0.5 or $5 \mathrm{mg} / \mathrm{kg}$ daily for 2 weeks) was effective at significantly improving ITPC in bilateral frontal areas and bilateral temporal areas of the brain, regions important for auditory stimulus processing, and cognitive response more generally. No effect was found in medial regions, indicating some region specificity of the TAK-063 effect on frontotemporal circuitry. This suggests that TAK-063 improves cortical auditory stimulus processing in Fmrl KO mice, without significantly depressing baseline EEG power or causing any noticeable sedation or behavioral side effects.

\section{Potential for TAK-063 in FXS Treatment}

In summary, TAK-063, a selective PDE10A inhibitor, has been tested in the standard mouse model of FXS (Fmrl KO mice). We found that TAK-063 ameliorates ITPC deficits in Fmrl KO mice, supporting its efficacy in improving a wellestablished EEG biomarker for FXS [18, 41]. Future studies 
will be required to evaluate the effects of TAK-063 on this important translational biomarker in FXS patients.

Supplementary Information The online version contains supplementary material available at https://doi.org/10.1007/s13311-021-01005-w.

Acknowledgments We acknowledge members of the Binder laboratory and Drs. Daniel Curran and Tauhid Ali from Takeda International for fruitful discussions. This work was supported by the Takeda International-UK, Rare Diseases Therapeutic Area Unit.

Required Author Forms Disclosure forms provided by the authors are available with the online version of this article.

\section{References}

1. Crawford DC, Acuña JM, Sherman SL. FMR1 and the fragile $X$ syndrome: Human genome epidemiology review. Genet Med, 3, 359-371 (2001).

2. Yu S, Pritchard M, Kremer E et al. Fragile X genotype characterized by an unstable region of DNA. Science, 252, 1179-1181 (1991)

3. Darnell Jennifer C, Van Driesche Sarah J, Zhang C et al. FMRP Stalls Ribosomal Translocation on mRNAs Linked to Synaptic Function and Autism. Cell, 146, 247-261 (2011).

4. Abbeduto L, Hagerman RJ. Language and communication in fragile X syndrome. Ment Retard Dev Disabil Res Rev, 3, 313-322 (1997).

5. Berry-Kravis E. Epilepsy in fragile X syndrome. Dev Med Child Neurol, 44, 724-728 (2002).

6. Hagerman RJ, Berry-Kravis E, Kaufmann WE et al. Advances in the treatment of fragile X syndrome. Pediatrics, 123, 378-390 (2009).

7. Miller LJ, McIntosh DN, McGrath J et al. Electrodermal responses to sensory stimuli in individuals with fragile $\mathrm{X}$ syndrome: A preliminary report. Am J Med Genet, 83, 268-279 (1999).

8. Musumeci SA, Hagerman RJ, Ferri R et al. Epilepsy and EEG Findings in Males with Fragile X Syndrome. Epilepsia, 40, 10921099 (1999).

9. Roberts JE, Hatton DD, Bailey DB. Development and Behavior of Male Toddlers With Fragile X Syndrome. J Early Interv, 24, 207223 (2001).

10. Sabaratnam M, Vroegop PG, Gangadharan SK. Epilepsy and EEG findings in 18 males with fragile $\mathrm{X}$ syndrome. Seizure, 10, 60-63 (2001)

11. Sinclair D, Oranje B, Razak KA, Siegel SJ, Schmid S. Sensory processing in autism spectrum disorders and Fragile $\mathrm{X}$ syndromeFrom the clinic to animal models. Neurosci Biobehav Rev, 76(Pt B), 235-253 (2017).

12. Van der Molen MJW, Huizinga M, Huizenga HM et al. Profiling Fragile X Syndrome in males: Strengths and weaknesses in cognitive abilities. Res Dev Disabil, 31, 426-439 (2010).

13. Wisniewski KE, Segan SM, Miezejeski CM, Sersen EA, Rudelli $\mathrm{RD}$. The fra(X) syndrome: Neurological, electrophysiological, and neuropathological abnormalities. Am J Med Genet, 38, 476-480 (1991).

14. Castrén M, Paakkonen A, Tarkka IM, Ryynanen M, Partanen J. Augmentation of auditory N1 in children with fragile X syndrome. Brain Topogr, 15(3), 165-171 (2003).

15. Schneider A, Leigh MJ, Adams $P$ et al. Electrocortical changes associated with minocycline treatment in fragile $\mathrm{X}$ syndrome. $\mathrm{J}$ Psychopharmacol, 27(10), 956-963 (2013).
16. Berry-Kravis EM, Lindemann L, Jønch AE et al. Drug development for neurodevelopmental disorders: lessons learned from fragile X syndrome. Nat Rev Drug Discov, 17, 280-299 (2018).

17. Jonak CR, Lovelace JW, Ethell IM, Razak KA, Binder DK. Reusable Multielectrode Array Technique for Electroencephalography in Awake Freely Moving Mice. Front Integr Neurosci, 12, 53 (2018).

18. Jonak CR, Lovelace JW, Ethell IM, Razak KA, Binder DK. Multielectrode array analysis of EEG biomarkers in a mouse model of Fragile X Syndrome. Neurobiol Dis, 138, 104794 (2020).

19. Ethridge LE, White SP, Mosconi MW et al. Neural synchronization deficits linked to cortical hyper-excitability and auditory hypersensitivity in fragile X syndrome. Mol Autism, 8, 22 (2017).

20. Kunitomo J, Yoshikawa M, Fushimi M et al. Discovery of 1-[2fluoro-4-(1H-pyrazol-1-yl)phenyl]-5-methoxy-3-(1-phenyl-1Hpyrazol-5-yl)pyri dazin-4(1H)-one (TAK-063), a highly potent, selective, and orally active phosphodiesterase 10A (PDE10A) inhibitor. J Med Chem, 57(22), 9627-9643 (2014).

21. Suzuki K, Harada A, Shiraishi E, Kimura H. In vivo pharmacological characterization of TAK-063, a potent and selective phosphodiesterase 10A inhibitor with antipsychotic-like activity in rodents. J Pharmacol Exp Ther, 352(3), 471-479 (2015).

22. Macek TA, McCue M, Dong X et al. A phase 2, randomized, placebo-controlled study of the efficacy and safety of TAK-063 in subjects with an acute exacerbation of schizophrenia. Schizophr Res, 204, 289-294 (2019).

23. Shiraishi E, Suzuki K, Harada A, Suzuki N, Kimura H. The Phosphodiesterase 10A Selective Inhibitor TAK-063 Improves Cognitive Functions Associated with Schizophrenia in Rodent Models. J Pharmacol Exp Ther, 356(3), 587-595 (2016).

24. Suzuki K, Harada A, Suzuki H, Miyamoto M, Kimura H. TAK063, a PDE10A Inhibitor with Balanced Activation of Direct and Indirect Pathways, Provides Potent Antipsychotic-Like Effects in Multiple Paradigms. Neuropsychopharmacology, 41(9), 22522262 (2016).

25. Suzuki K, Kimura H. TAK-063, a novel PDE10A inhibitor with balanced activation of direct and indirect pathways, provides a unique opportunity for the treatment of schizophrenia. CNS Neurosci Ther, 24(7), 604-614 (2018).

26. Harada A, Suzuki K, Kimura H. TAK-063, a Novel Phosphodiesterase 10A Inhibitor, Protects from Striatal Neurodegeneration and Ameliorates Behavioral Deficits in the R6/2 Mouse Model of Huntington's Disease. J Pharmacol Exp Ther, 360(1), 75-83 (2017).

27. Berry-Kravis E, Hicar M, Ciurlionis R. Reduced cyclic AMP production in fragile $\mathrm{X}$ syndrome: cytogenetic and molecular correlations. Pediatr Res, 38(5), 638-643 (1995).

28. Berry-Kravis E, Huttenlocher PR. Cyclic AMP metabolism in fragile X syndrome. Ann Neurol, 31(1), 22-26 (1992).

29. Maurin T, Melancia $F$, Jarjat $M$ et al. Involvement of Phosphodiesterase 2A Activity in the Pathophysiology of Fragile X Syndrome. Cereb Cortex, 29(8), 3241-3252 (2019).

30. Choi CH, Schoenfeld BP, Weisz ED et al. PDE-4 inhibition rescues aberrant synaptic plasticity in Drosophila and mouse models of fragile X syndrome. J Neurosci, 35(1), 396-408 (2015).

31. Gurney ME, Cogram P, Deacon RM, Rex C, Tranfaglia M. Multiple Behavior Phenotypes of the Fragile-X Syndrome Mouse Model Respond to Chronic Inhibition of Phosphodiesterase-4D (PDE4D). Sci Rep, 7(1), 14653 (2017).

32. Bakker CE, Verheij $\mathrm{C}$ et al. Fmr1 knockout mice: a model to study fragile X mental retardation. The Dutch-Belgian Fragile X Consortium. Cell, 78(1), 23-33 (1994).

33. Artieda J, Valencia M, Alegre M, Olaziregi O, Urrestarazu E, Iriarte J. Potentials evoked by chirp-modulated tones: A new technique to evaluate oscillatory activity in the auditory pathway. Clin Neurophysiol, 115, 699-709 (2004). 
34. Pérez-Alcázar M, Nicolás MJJ, Valencia M, Alegre M, Iriarte J, Artieda J. Chirp-evoked potentials in the awake and anesthetized rat. A procedure to assess changes in cortical oscillatory activityExp Neurol, 210, 144-153 (2008).

35. Purcell DW, John SM, Schneider BA, Picton TW. Human temporal auditory acuity as assessed by envelope following responses. $\mathrm{J}$ Acoust Soc Am, 116, 3581-3593 (2004).

36. Tallon-Baudry C, Bertrand O, Delpuech C, Pernier J. Stimulus specificity of phase-locked and non-phase-locked $40 \mathrm{~Hz}$ visual responses in human. J Neurosci, 16, 4240-4249 (1996).

37. Maris E, Oostenveld R. Nonparametric statistical testing of EEGand MEG-data. J Neurosci Methods, 164(1), 177-190 (2007).

38. Berry-Kravis E, Sklena P. Demonstration of abnormal cyclic AMP production in platelets from patients with fragile X syndrome. Am J Med Genet, 45(1), 81-87 (1993).
39. Tsai M, Chrones L, Xie J, Gevorkyan H, Macek TA. A phase 1 study of the safety, tolerability, pharmacokinetics, and pharmacodynamics of TAK-063, a selective PDE10A inhibitor. Psychopharmacology, 233(21-22), 3787-3795 (2016).

40. Sinclair D, Featherstone R, Naschek $M$ et al. GABA-B Agonist Baclofen Normalizes Auditory-Evoked Neural Oscillations and Behavioral Deficits in the Fmrl Knockout Mouse Model of Fragile X Syndrome. Eneuro, 4, ENEURO.0380-0316.2017 (2017).

41. Ethridge LE, De Stefano LA, Schmitt LM et al. Auditory EEG Biomarkers in Fragile X Syndrome: Clinical Relevance. Front Integr Neurosci, 13, 60 (2019).

Publisher's Note Springer Nature remains neutral with regard to jurisdictional claims in published maps and institutional affiliations. 